\title{
PRESERVAR LO INTANGIBLE: El caso de los polígonos de Casas Baratas de Barcelona.*
}

\author{
Núria NOGUER PUJADAS / Adolf SOTOCA GARCIA
}

La actual transformación del barrio de las casas baratas del Bon Pastor constituye uno más de los múltiples procesos de renovación urbana que han suscitado un polémico debate en la ciudad de Barcelona. La dialéctica entre mejora urbana y preservación del patrimonio (físico, histórico y social) se encuentra en el fondo del debate. Los argumentos a favor de la protección del patrimonio físico se han basado frecuentemente en la singularidad arquitectónica o en el valor tipológico de tejidos. En los polígonos de Casas Baratas en Barcelona, la preservación no puede defenderse desde la atención a los objetos arquitectónicos singulares. Los barrios de Casas Baratas constituyen, sin embargo, conjuntos de gran valor desde el punto de vista tipológico: su morfología urbana, además de ser testimonio vivo de un momento histórico, ha propiciado usos sociales del espacio público muy particulares. Este escrito argumenta a favor de su valorización morfológica. Y lo hace describiendo su encaje en los tres procesos de transformación ya culminados, con el objetivo de obtener criterios para intervenciones futuras en las que actualización y preservación no sean necesariamente excluyentes.

Conservación urbana, sustitución urbana, patrimonio urbano.

\section{PRESERVING THE INTANGIBLE: The case of Barcelona's cheap houses polygons.}

The ongoing urban renewal of "Casas Baratas" of Bon Pastor is one of the several processes of transformation that have raised a controversial debate in the city of Barcelona. The dialectic between urban renewal and heritage preservations (physical, historical and social) is in the very core of the debate. The reasons to preserve the physical heritage have been often based on the architectural uniqueness or the typological value of urban fabric. In the "Casas Baratas" states of Barcelona, preservation cannot be defended for having singular architecture; however, from a typological point of view, they constitute very interesting urban fragments. Besides their great value as living heritage of a certain historic period of social housing in Barcelona, their particular morphology stimulated specific social uses of public space. This paper states their morphological value. And, by analysing the completed urban renewal processes, we aim to infer criteria for future interventions where renewal and preservation won't be necessarily incompatible.

Urban preservation, urban replacement, urban beritage. 


\section{De la construcción a la obsolescencia: tres momentos en cincuenta ańos.}

Desde su construcción en 1929 hasta la constitución de los ayuntamientos democráticos en 1979 los polígonos de Casas Baratas en Barcelona padecieron una evolución histórica que explica su razón de ser actual y la necesidad de plantear de forma crítica su renovación. Naturalmente, las condiciones de los cuatro polígonos de Casas Baratas son desiguales, como lo son sus procesos de transformación. No obstante, en todos ellos las razones de degradación física y social a lo largo del tiempo cabe encontrarlas en la gestión y opciones tomadas ya en los proyectos de 1929. Posteriormente la consideración negativa que de los polígonos hacen los planes de 1953 y 1976 acaba por deteriorar el entorno físico y social de estos barrios, situándolos como espacios de renovación prioritaria en las políticas urbanas de la ciudad.

\section{Los polígonos en 1929: accesibilidad y encaje urbano.}

La construcción de los barrios de Casas Baratas en Barcelona es la respuesta que el Ayuntamiento dio al acuciante problema de la vivienda obrera de los años 20. Barcelona recibe en estos años un índice muy elevado de inmigrantes como consecuencia de la fuerte demanda de mano de obra industrial. Las grandes obras públicas como el Gran Metropolitano o el Metro Transversal por un lado, y la Exposición Universal del 1929 por el otro, son las razones del aumento del ritmo migratorio.

El Marco legislativo en el cual se enmarcaron estas primeras operaciones en política de vivienda pública condicionó enormemente el margen de actuación municipal. Por un lado, atribuía a las casas baratas un papel puramente asistencial y transitorio al prever su sustitución en caso de incremento de las plusvalías de sus entornos urbanos próximos. ${ }^{1}$ Por otro lado, y después de múltiples modificaciones, el RD de 10 de octubre de 1924 restringe el ámbito de actuación municipal a la promoción de nuevos barrios periféricos alejados de las zonas centrales de la ciudad. Por ello el Ayuntamiento adquirió, mediante el Patronato de la Habitación de Bar-

\footnotetext{
* Artículo anteriormente publicado como: "Casas Baratas": replacement or preservation. Proceedings or magazine (name, volume, pages): CAH 20thC: Intervention approaches for the 20th century architectural heritage, Madrid, Ministerio de Cultura, 2011. Year:2011

1 "Cuando el crecimiento de la ciudad y el alquiler de las propiedades vecinas haya crecido hasta el punto de hacer irrisorio el de las casas baratas, se procederá a su venta y a su sustitución por otras casas baratas de igual clase en lugares oportunos", Informe de política de vivienda, 1920. En "Barcelona, les Cases Barates"
} 
celona, terrenos en cuatro emplazamientos diferentes, el único denominador común de los cuales era su bajo coste. Atendiendo a este criterio, los terrenos escogidos se encontraban en posiciones marginales con respecto a la ciudad central, tanto en lo que refiere a la accesibilidad en transporte público, como a la continuidad del tejido urbano con las tramas vecinas. Los barrios finalmente construidos son los polígonos de "Baró de Viver", "Milans del Bosch" - posteriormente conocido como Bon Pastor, "Eduard Aunòs" y "Ramón Albó" actualmente conocido como el barrio de "Can Peguera"

El redibujo de la cartografía levantada por Vicenç Martorell en los años 30 (Fig.1a) muestra la posición de aislamiento de tres de los conjuntos con respecto a las tramas urbanas consolidadas. El único de ellos que en sus orígenes goza de una continuidad con los tejidos urbanos existentes es el grupo de Ramon Albó. El Paseo Urrútia, límite norte del barrio, ha sido desde los ańos 30 una vía estructural de Barcelona mientras que las calles Congreso y Feliu Codina comunicaban el barrio con el casco antiguo de Horta. El análisis a una escala más detallada (Fig. 1b) muestra el contraste entre este conjunto y el resto de barrios de Casas Baratas, situados en entornos escasamente urbanos y en los que dominan los usos rurales del suelo.

Por lo que respecta al acceso en transporte público los emplazamientos para tres de los polígonos planificados se situaban a más de media hora caminando respecto a la línea de tranvía más próxima. Este era el caso para los conjuntos de Milans del Bosch y Baró de Viver, a media hora de la parada más próxima del tranvía 40, y para el conjunto de Eduard Aunós, cuya conexión con la ciudad central se produjo sólo a partir de 1939 mediante la línea 48. Únicamente el conjunto de viviendas Ramon Albó se encontraba razonablemente bien conectado mediante la línea $46,{ }^{2}$ cuya parada en la Plaza Ibiza se encontraba a menos de un cuarto de hora del barrio. Así este barrio goza, también, de mejores condiciones que el resto de conjuntos.

\section{El Plan Comarcal de 1953: aislamiento y marginalidad.}

Con la aprobación del Plan Comarcal de 1953 se podría haber dado por primera vez una visión integradora de los polígonos de casas baratas en un contexto metropolitano complejo. A nivel estructural el Plan preveía un sistema policéntrico de núcleos históricos que actuaban como centralidades urbanas del resto de barrios residenciales. En los intersticios y suelo sobrante, se sitúan áreas industriales, zonas verdes e infraestructuras de comunicación. El Plan delimitaba extensas áreas de industria en

2 El tranvía eléctrico de Barcelona a Horta, en "Destinació: Nou Barris, un viatge per la historia dels transports a Nou Barris" y "Els tramvies de Barcelona (de 1929 ençà) Història i explotació" 


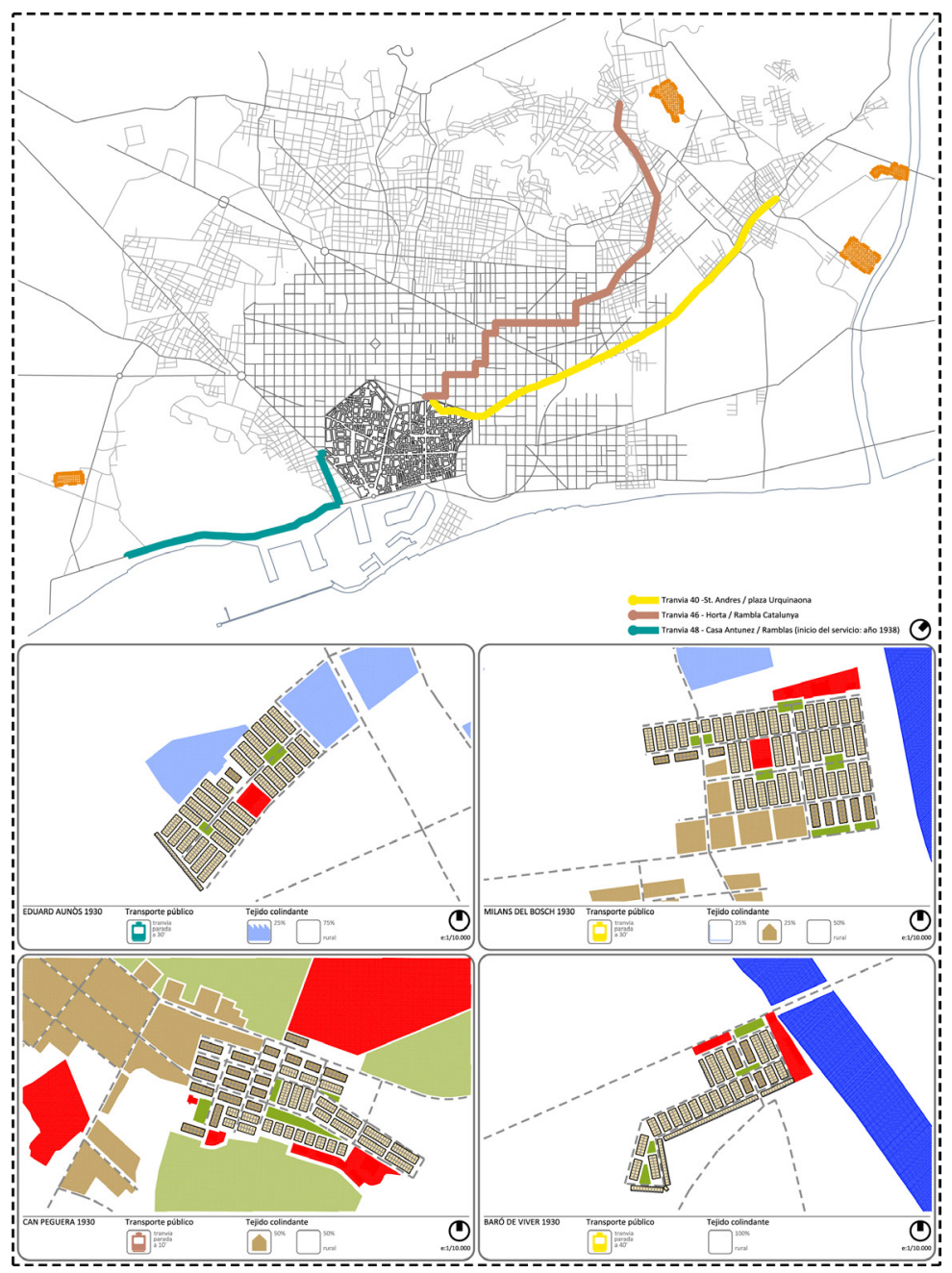

Fig.1: Encaje urbano de los polígonos de casas Baratas en 1930, localización y accesibilidad en transporte público. Aproximación, tejidos colindantes. 


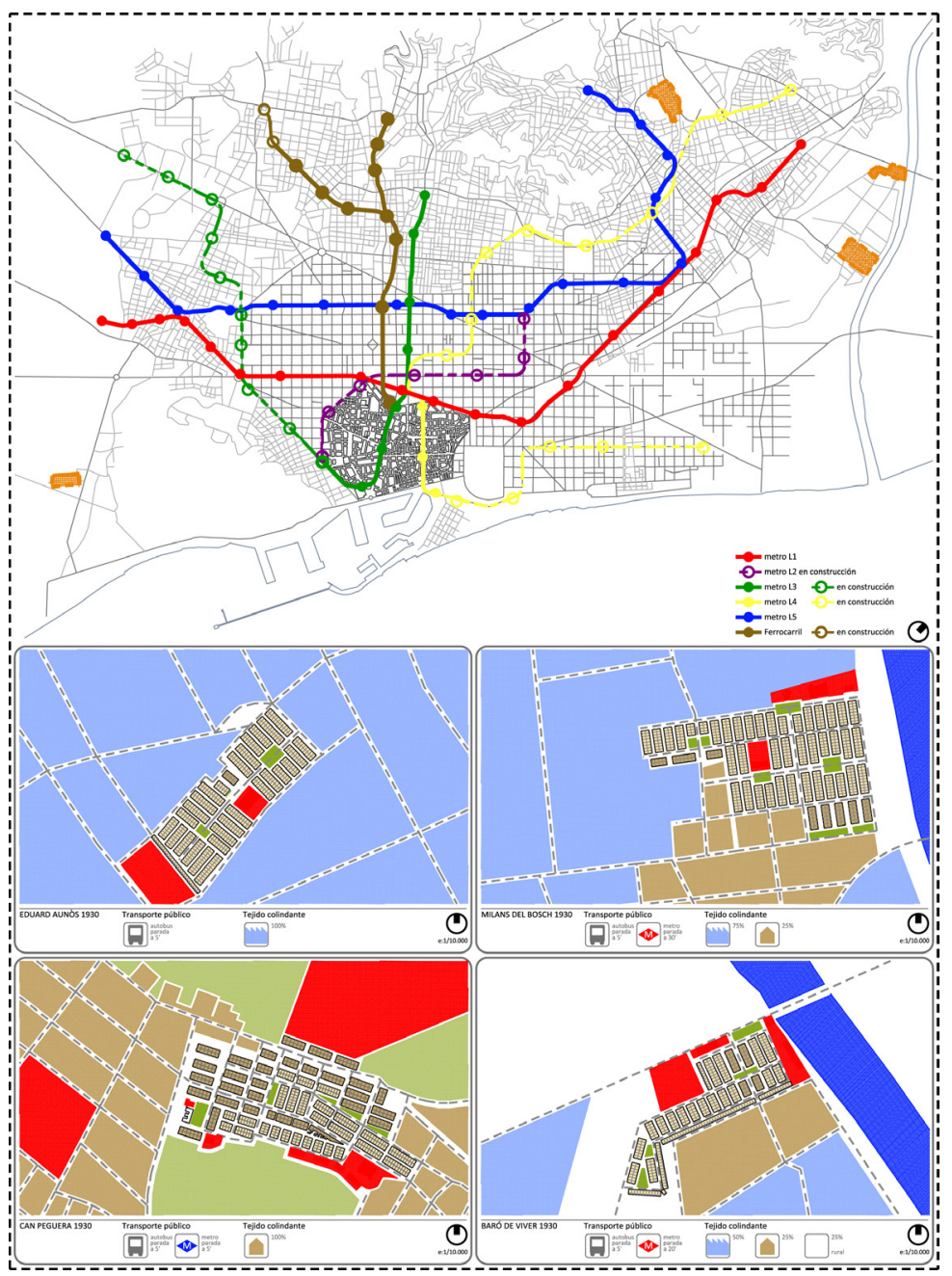

Fig.2: Encaje urbano de los polígonos de casas Baratas en 1970, localización y accesibilidad en transporte público. Aproximación, tejidos colindantes. 
todo el Levante barcelonés, dónde se encontraban los polígonos de "Baró de Viver" y "Milans del Bosch" y también en toda la zona portuaria y sus proximidades, dónde se encontraba el polígono de "Eduard Aunós". Consecuentemente, estos tres barrios quedaron rodeados de suelo para grandes y pequeñas industrias incompatibles con los usos residenciales. La rápida industrialización de Barcelona provocó una intensa urbanización industrial en estos ámbitos a lo largo de los años 60, tal y como podemos observar en el redibujo del ortofotoplano del año 1970 (Fig. 2b)

De nuevo las condiciones del conjunto Ramon Albó son más favorables que el resto de polígonos de Casas Baratas. El Plan, además de calificar como parque urbano al cercano Turó de la Peira, reconoce las tramas residenciales existentes facilitando de esta manera la integración del conjunto con el entorno urbano próximo. Con la aprobación del Plan Parcial residencial del Turó de la Peira en 1959 se consolida esta relación, ya que el nuevo tejido residencial construido posibilitó la apertura de nuevos ejes viarios que relacionaron el conjunto de casas baratas con la estructura principal de la ciudad a través del Paseo Maragall.

\section{El Plan General Metropolitano de 1976: la sustitución como camino.}

El PGM de 1976 coincidió con el final de la dictadura y con el emergente protagonismo de las reivindicaciones vecinales en los procesos de planificación urbana. Las determinaciones del Plan se orientaron hacia el control de los aprovechamientos urbanísticos, la dotación de la ciudad de nuevos equipamientos y la atención sobre la mejora de tejidos urbanos degradados. Definido este marco de actuación, el Plan asienta las bases para la futura transformación de los cuatro polígonos de Casas Baratas. A pesar de que en un inicio el Plan califica de forma diferenciada cada uno de los barrios, ${ }^{3}$ la ejecución del mismo ha resultado en la transformación urbana de tres de ellos y una cierta indefinición urbanística que, aún a día de hoy, prevalece en el conjunto de casas Ramón Albó.

Los conjuntos de Baró de Viver y Eduard Aunós han completado ya su transformación a partir de sendos documentos urbanísticos aprobados en 1991 y 1995 respectivamente. El conjunto de Milans del Bosch, por su parte, está a día de hoy en pleno proceso de sustitución física, de acuerdo con las determinaciones de la modificación de PGM aprobada en 2002. Nos encontramos, por tanto, ante un tema de actualidad pero del cual ya tenemos una notable experiencia acumulada. Del estudio y análisis de esta

3 El Plan General Metropolitano califica como zona de remodelación pública -clave14a- el polígono de Ramón Albó y como zona de remodelación privada -clave 14b- el polígono de Baró de Viver. Se genera de esta manera, en virtud de las determinaciones de la clave 14 del PGM, el marco jurídico que avala la posterior sustitución de ambos conjuntos. En el caso de los polígonos de Milans del Bosch y Eduard Aunós el Plan General reconoce la sujeción a ordenación volumétrica anterior -clave 18-. Sin embargo, dado que ambos conjuntos habían sido incluidos con anterioridad en sendos Planes Parciales que preveían su sustitución, la calificación como zona 18 abrió la puerta, en virtud de lo establecido por el artículo 336 de la normativa del PGM, a su posterior sustitución. 
experiencia pueden extraerse, sin duda, criterios proyectuales que pauten una más consciente actitud respecto a decisiones que, respecto al conjunto de Ramón Albó, deberán tomarse en un futuro no muy lejano.

\section{Sobre oportunidades perdidas: la sustitución de las Casas Baratas}

Con la constitución de los ayuntamientos democráticos en 1979 el nuevo Patronato Municipal de la Vivienda de Barcelona, propietario y responsable de la gestión de los polígonos de Casas Baratas, establece estrategias con el objetivo de dignificar el parque inmobiliario que tenía en régimen de alquiler. Durante los primeros años se emprendieron programas de rehabilitación y mejora de las viviendas. Pronto se decidió, no obstante, la sustitución de estos conjuntos. Difícilmente puede ponerse en duda esta decisión, a la vista de las condiciones objetivas de deterioro físico de los conjuntos y de la difícil adaptación de las unidades residenciales a los estándares de habitabilidad. Sí que parece pertinente, en cambio, evaluar el modo como este reemplazo se ha completado en relación con el espacio urbano y el valor tipológico y morfológico de estos barrios.

Cada uno de los procesos de renovación que sobre los polígonos de Casas Baratas han tenido lugar presenta sus propias especificidades. A pesar de ello todos pueden ser analizados en relación a sus estrategias respecto tres líneas de actuación claves en cualquier proceso de reforma urbana: las dotaciones y espacios libres, la continuidad con respecto las tramas urbanas vecinas y la mejora o sustitución del parque inmobiliario existente. Asumiendo que cualquier acción transformadora sobre estos barrios hubiera debido considerar la preservación de la identidad morfológica de cada conjunto, podemos analizar cada línea de actuación desde el justo equilibrio entre preservación física y necesidad de actualización.

\section{Espacios libres y equipamientos, testimonios de la esfera colectiva.}

Los proyectos originales de cada uno de los polígonos de casas baratas preveía la construcción de un pequeño equipamiento comunitario. Se trata en todos los casos de un grupo escolar dimensionado para 600-800 alumnos. Los proyectos para los grupos de Eduard Aunós y Ramón Albó preveían, además, la construcción de un cuartel de la Guardia Civil. Por otro lado, cada uno de los barrios incluye, aunque de manera tímida, un elemento de espacio público que, en forma de paseo arbolado, adquiere un cierto valor significativo en el conjunto. Ambos elementos, espacio libre y equipamiento, aunque no siempre concebidos de manera sistémica y coherente entre sí, se consolidaron con el tiempo como el escenario de la esfera colectiva en estos barrios.

Con el paso del tiempo la precaria dotación de equipamientos en los polígonos de Casas Baratas se ha ido completando. La gradual transforma- 


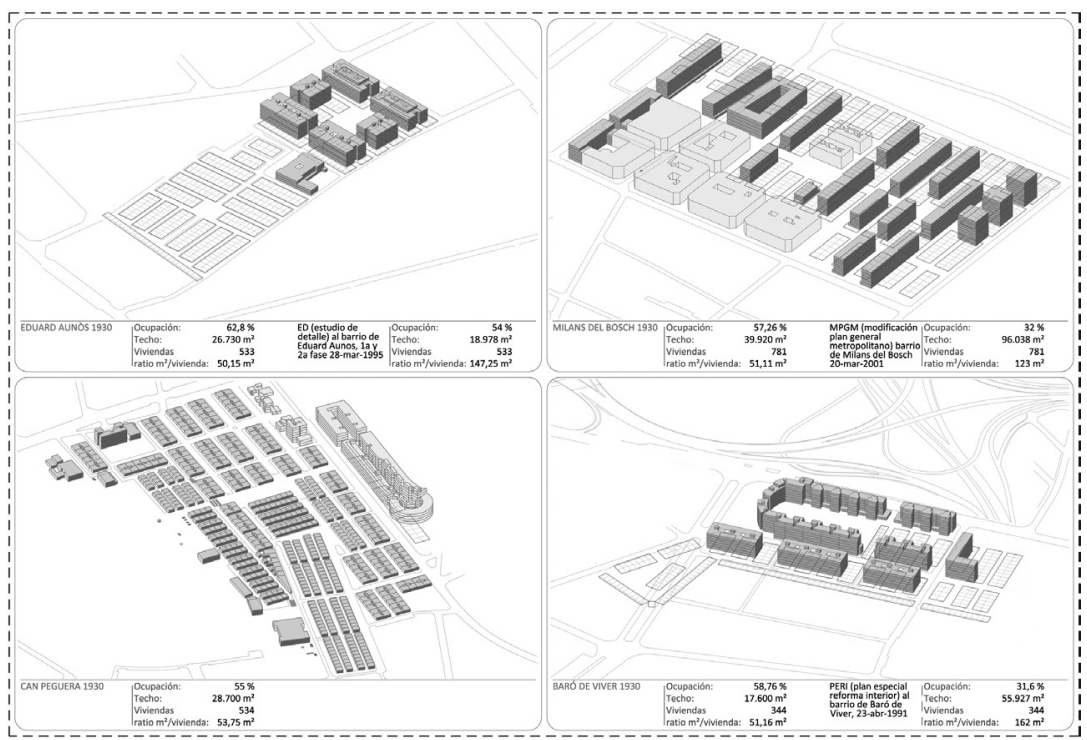

Fig.3: Los procesos de renovación y sustitución; superposición de la trama original 1930 con los resultados de los distintos procesos.

ción de los tejidos próximos ha significado una sustantiva construcción de equipamientos públicos en un área de proximidad más que razonable. ${ }^{4} \mathrm{La}$ construcción de nuevos equipamientos no ha sido, por tanto, un objetivo prioritario de las operaciones de transformación sobre unos barrios ya suficientemente dotados. La discusión, en cambio, sí que hubiera podido establecerse en relación al papel que pueden jugar los grupos escolares construidos en 1929. A pesar de su objetivo escaso valor arquitectónico estos edificios hubiesen podido ser, por su singularidad dentro del conjunto y por su lugar en la memoria de los residentes, elementos garantes de una cierta identidad colectiva. La transformación de los conjuntos de Eduard Aunós y Baró de Viver no mantuvieron, sin embargo, estas edificaciones. En el caso de Milans del Bosch, aunque se mantiene físicamente el edificio, no se le dota de contenido significativo dentro del proyecto.

Por lo que refiere a los ejes de espacio libre de cada conjunto, bien puede argumentarse en el mismo sentido. La posición que estos elementos ocupan dentro de la ordenación general de cada conjunto les otorga un papel

4 A pesar de que estas nuevas dotaciones no se encuentran dentro del los polígonos de Casas Baratas, se han dado en sus proximidades grandes mejoras en políticas de dotaciones públicas, especialmente en los barrios de Milans del Bosch y Can Peguera. Las nuevas dotaciones en el conjunto Eduard Aunòs son una parroquia y campo de fútbol municipal; en Milans del Bosch el complejo deportivo municipal, un centro de atención Primaria, un mercado, una parroquia, una biblioteca pública, un centro de servicios sociales, y varios centros docentes; en Baró de Viver una parroquia, instalaciones deportivas municipales y varios centros docentes; finalmente, en Can Peguera se han construido en el periodo democrático una parroquia, un centro cívico, huertos urbanos, una escuela taller, un casal de barrio, residencias para gente mayor, un centro de asistencia sanitaria primaria, diversas instalaciones deportivas, una biblioteca y varios centros docentes. 
estructurador en el barrio que bien hubiese merecido ser contemplado en los procesos de transformación. El incremento de zona verde a la que toda transformación se ve obligada ${ }^{5}$ hubiese sido, además, una excelente oportunidad para reforzar los elementos de espacio público existente y mejorar su relación con los equipamientos originales de los conjuntos.

En lugar de ello, los sistemas de espacios libres propuestos en la renovación de Milans del Bosch y Baró de Viver se desvinculan de los conjuntos originales incorporando lógicas de ordenación ajenas a la memoria del lugar (Fig. 3). En el caso del grupo Eduard Aunós, la nueva ordenación de los espacios libres recuerda, caso de haberse completado las dos fases del proyecto, a la estructura del conjunto original. No obstante, la construcción de edificios de $\mathrm{PB}+5$ en lugar de las casas en planta baja originales desvirtúa la proporción del espacio libre original. No sólo la geometría en planta, sino también su relación volumétrica forma parte de la identidad del espacio público en estos conjuntos.

\section{Integración urbana y programación temporal.}

La aprobación del PGM en 1976 dejó asentadas las bases para la intervención en los barrios de Casas Baratas. El Plan no proponía cambios sustanciales en sus entornos próximos y, por ello, las operaciones fueron entendidas parcialmente dentro de cada uno de las piezas. Se perdieron de esta manera las oportunidades que hubiesen podido significar su integración en proyectos más ambiciosos para ámbitos más extensos de la ciudad. Los procesos de renovación en estos barrios no han dialogado con las transformaciones que, antes o después, han tenido lugar en ámbitos vecinos. ${ }^{6}$ Por ello, con frecuencia, las tramas viarias resultantes de la transformación son inconexas y faltas de coherencia con los tejidos vecinos, persistiendo de esta manera el original aislamiento de estas piezas con su entorno urbano inmediato.

Una más coherente programación hubiese podido mejorar notablemente la integración y cohesión de los tejidos urbanos resultantes. Puede decirse, sin embargo, que los planes de transformación sí se programan en relación

5 El artículo 94 de la LU establece la necesidad de incrementar la reserva de zona verde en determinadas operaciones de mejora urbana. Literalmente establece: "Si la modificación de una figura del planeamiento urbanístico supone un incremento de techo edificable, en el caso de suelo urbanizable, se incrementarán proporcionalmente, como mínimo, los espacios libres y las reservas para equipamientos determinados por el artículo 65.3 y 4 , y, en el caso de suelo urbano, debe preverse una reserva mínima para zonas verdes y espacios libres públicos de $20 \mathrm{~m} 2$ por cada $100 \mathrm{~m} 2$ de techo residencial y de $5 \mathrm{~m} 2$ por cada $100 \mathrm{~m} 2$ de techo de otros usos. Para computar estos estándares, se aplicará lo establecido en el artículo 65.5. Además, si es necesario, se debe reservar suelo para equipamientos públicos en la cantidad adecuada para hacer frente a los requerimientos que derivan de la modificación".

6 Por ejemplo, todo el sector del levante Barcelonés, dónde se encuentran los polígonos de Milans del Bosch y Baró de Viver, se está transformando considerablemente, sobre todo a raíz del Plan Sagrera, que limita al Nord-este con Baró de Viver, o la pendiente transformación de uso del polígono industrial del Bon Pastor. En el caso de Eduard Aunos, nos encontramos con la misma situación: el tejido industrial colindante, está pendiente de transformarse, tras la aprobación definitiva el 1 de junio de 2006 de la Modificación del Plan General Metropolitano para la transformación urbanística de la Marina de la Zona Franca. 
a las mejoras de accesibilidad en transporte público: la llegada del metro a estos barrios ha sido en todos los casos el catalizador de las operaciones de renovación urbana. ${ }^{7}$ La integración de los polígonos de Casas Baratas en la Barcelona metropolitana ha sido, por tanto, más funcional que morfológica.

\section{Tipología y uso social del espacio urbano}

La actualización del parque inmobiliario fue, ya desde 1982, una acción prioritaria que justificó y dio sentido a los procesos de sustitución de los polígonos de Baró de Viver, Eduard Aunós y, posteriormente, de Milans del Bosch. Más allá de las patologías físicas que comprometían incluso las más elementales condiciones de habitabilidad, la tipología residencial dominante en estos conjuntos hacía difícil, por sus dimensiones, la viabilidad de estos barrios como entornos habitables.

Las viviendas construidas por el Patronato de la Habitación en los Grupos de Casas Baratas se agrupan según dos tipos arquitectónicos elementales: el tipo A, con una superficie edificada de $61,15 \mathrm{~m} 2$ en una parcel.la de $80 \mathrm{~m}^{2}$ consta de cuatro habitaciones, cocina, patio cubierto con lavabo y WC, lavadero, y un reducido espacio descubierto. Estos tipos de unidades poseen un pequeño jardín en la parte anterior. Las viviendas del tipo B, con una superficie construida de unos $50 \mathrm{~m} 2$, constan de tres habitaciones, cocina, patio cubierto con WC y lavabo, lavadero y un pequeño espacio descubierto. A excepción del conjunto de Ramón Albó, en el que conviven de forma equitativa ambos tipos, predomina en los otros tres barrios el de más reducidas dimensiones. Las viviendas del tipo B significan un $90 \%$ sobre el total en Milans del Bosch y Baró de Viver y un 98,5\% en el barrio de Eduard Aunòs. Puede afirmarse, por tanto, que en estos tres conjuntos, a una tipología de muy escasa superficie, se le suma una enorme monotonía tipológica. De nuevo, las condiciones en el polígono de Ramón Albó son claramente más favorables.

La sustitución de las viviendas ha significado un notable incremento cuantitativo de la superficie media por vivienda (Fig. 3). Se ha pasado de unidades de 50 o $60 \mathrm{~m} 2$ de techo construido a viviendas con una superficie construida media de 109 m2 -Baró de Viver-, 82 m2 -Eduard Aunós- y $101 \mathrm{~m} 2$-Milans del Bosch-.

7 En el Barrio de Baró de Viver, se inaugura la parada de Metro el Diciembre del año 1983 y los primeros documentos de avance para el plan de reforma interior del Barrio datan de 1987. La estación de Metro de Bon Pastor (L9 y L10), se puso en marcha el 18 de Abril de 2010 aunque el proyecto de construcción de la línea 9 comenzó en el Plan director de Infraestructuras 2001-2010 (Pla director d'Infraestructures 2001-2010), que fue aprobado por la Autoridad del Transporte Metropolitano el 25 de abril de 2002. La Modificación del Plan General Metropolitano para el barrio del Bon Pastor es de 2001. Eduard Aunòs, al estar dentro del nuevo barrio residencial la Marina de la Zona Franca verá mejorada su accesibilidad, ya que el proyecto del barrio incluye la construcción de dos estaciones de metro correspondientes a la línea 2 (ampliación de la línea) y la línea 9 que conectaran el barrio en quince minutos con el centro de la ciudad. 
En lo que a modificación cualitativa del espacio urbano se refiere, merece la pena destacar que en los tres casos se ha optado como solución tipológica por el bloque plurifamiliar de entre 5 y 8 plantas. De esta manera la ordenación resultante puede asumir el incremento de techo derivado de la ampliación del tamaño medio de las viviendas. Esta opción proyectual ha modificado decisivamente la forma urbana de los conjuntos, incidiendo en el uso social del espacio libre. Si la ocupación media de la edificación en los proyectos originales se situaba sobre el $60 \%$, las edificaciones resultantes de los procesos de sustitución ocupan el 25-33\% de la superficie total del ámbito (Fig. 3) En el caso del PERI Baró de Viver, la ocupación se sitúa incluso por debajo del 15\%. Este tipo de ordenación, poco extensiva y concentrada en altura, incidirá decisivamente en el uso social que del espacio urbano se hace. De unos proyectos originales en los que la relación entre la vivienda en planta baja y el espacio urbano se establece de forma natural, se pasa a un tipo de ordenación en el que el espacio urbano y vivienda acaban articulándose como realidades autónomas y de difícil relación. Si a ello le añadimos la modificación drástica que las sustituciones han significado en términos de sistemas de espacios libres y equipamientos, puede concluirse que se ha dado en estos procesos una evidente pérdida de la identidad urbana de los conjuntos.

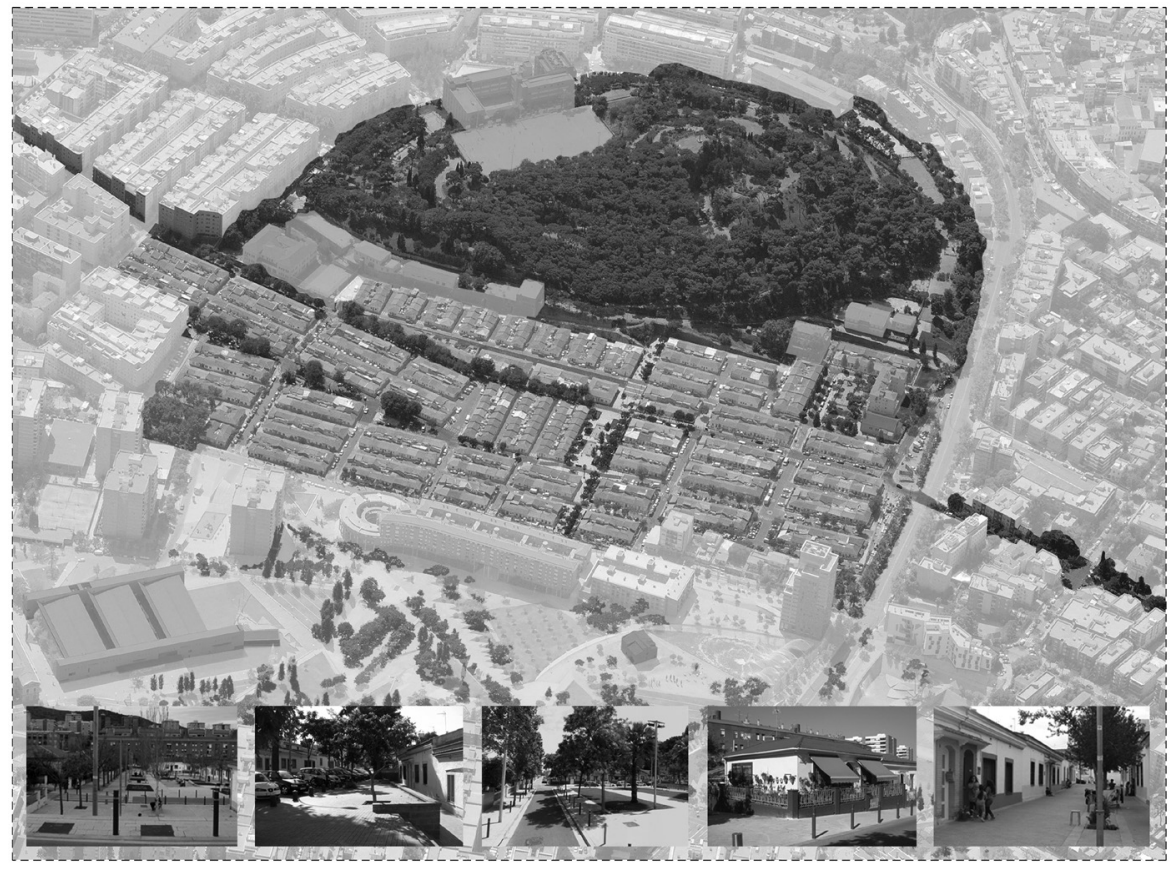

Fig.4: Barrio de Can Peguera, estado actual y criterios de intervención 


\section{Can Peguera. Un espacio a preservar}

El barrio de Can Peguera -Ramón Albó-, es el único que hoy, después de las diversas intervenciones llevadas a cabo en los polígonos de Casas Baratas, permanece con los mismos atributos que el proyecto original. A lo largo del artículo hemos podido ver como esta pervivencia no ha sido una casualidad; de él, hemos podido destacar características que lo sitúan en mejores condiciones que el resto de polígonos.

Desde su construcción en el año 1929, la localización del barrio y su encaje dentro de la ciudad ha sido más favorable, tal y como hemos visto reflejado en las Fig. 1 y 2. Las tramas urbanas vecinas, de uso residencial, se han completado y cohesionado con los años. Ejes como el Aneto-Vilaseca-Congrès que atraviesa el barrio de este a oeste o el eje Darnius (Fig. 4a) que conecta dos grandes parques pasando por el centro del barrio en sentido norte-sud, ponen en relación el barrio con su entorno y con la ciudad. La integración urbana del conjunto ha propiciado con los años la consolidación y rehabilitación de la estructura original de equipamientos y espacios libre. De este modo, los espacios de la esfera colectiva no han sido modificados con el paso del tiempo sino que se han ido actualizando en función de los requerimientos de cada momento histórico. Por un lado, la calle Darnius (Fig. 4a) ha sido urbanizada recientemente, reforzando así su rol como eje cívico del barrio. Por el otro, el antiguo cuartel de la Guardia Civil y el centro escolar, funcionan a día de hoy como casal para gente mayor con huertos urbanos y como escuela taller con casal de barrio respectivamente. Desde el punto de vista de las unidades-vivienda, Can Peguera es, además, el único barrio con una cierta diversidad tipológica y con una real aptitud para adaptarse a las condiciones de habitabilidad exigibles a día de hoy. La implicación de los residentes y las constantes obras de mejora que se han emprendido en el barrio como resultado de su iniciativa nos hablan, además, de un arraigo social cierto.

Este núcleo de casas baratas consta en el Plan General Metropolitano como uno de los afectados por un proceso remodelación. Los derribos de los grupos Eduard Aunós, Baró de Viver y Milans del Bosch han situado a Can Peguera como el último testimonio de un momento clave en la historia y de una época en la construcción de la vivienda obrera en Barcelona. Por este motivo, además de por su valor morfológico y social, debe ser conservado y preservado. Ello no significa renunciar a su actualización, por otro lado necesaria. Considerar los ejes de espacio público originales (en rojo Fig.4) e integrar el conjunto en las tramas urbanas vecinas a través de nuevas calles peatonales (Riells-Espigua y Montagut en verde Fig.4) parecen opciones más que adecuadas. La sustitución de piezas parece igualmente pertinente, sobre todo para las unidades-vivienda con graves patologías o de reducidas dimensiones. Pero dicha sustitución no debe implicar, en ningún caso, la alteración del orden morfológico del conjunto pues éste es, precisamente, su valor. Los 
elementos estructurales deben pautar la toma de decisiones acertadas, considerando al barrio no únicamente como la suma de sus viviendas, sino como tejido urbano complejo, rico y, sobretodo, vivo.

\section{Referencias bibliográficas:}

AA.VV, Generalitat de Catalunya: "Reviure els barris : programes de nous habitatges de substitució per a la millora de les àrees urbanes de Catalunya". Generalitat de Catalunya. Departamento de Politica Territorial y Obras Publicas, 2001.

AA. VV, Grupo de historia Nou Barris-Can Basté, "Del despertar al trencament. Nou Barris 1897-1936", Ayuntamiento de Barcelona, Distrito de Nou Barris,

AA.VV, Patronato Municipal de la Vivienda, "'De les cases barates als grans polígons, el Patronat Municipal de Barcelona entre 1929 i 1979", Ayuntamiento de Barcelona, 2003.

Domingo, Miquel; Segarra, Ferran, "Barcelona: Les cases Barates”, Ayuntamiento de Barcelona, 1999.

Garcia Castro de la Peña, Teresa, "Barrios Barceloneses de la dictadura de Primo de Rivera", Tesis doctoral dirigida por el Dr. Horacio Capel Sáez y presentada en el Departamento de Geografía de la Universidad de Barcelona, 1973, pag.77-94

Gonzàlez, Albert, "Els tramvies de Barcelona (de 1929 ençà) Història i explotacó", Colección "camí ral" ํ11, Barcelona, Rafael Dalmau Editor, 1998.

Fernandez, Ricard, "Destinació: Nou Barris, un viatge per la història de Nou barris", Ayunatmiento de barcelona, 2010.

Ferrer, Amador, "Els Polígons de Barcelona : l'habitatge massiu i la formació de l'àrea metropolitana", Barcelona, Ediciones UPC, 1996.

Fabre, Jaume; Huertas, Josep Maria, “Tots els barris de Barcelona”, Barcelona, Ediciones 62, 1977.

Oyón, José Luís, "La conservación de la vivienda popular en las periferias urbanas. El caso de BarceIona y la colonia castells" Scripta Nova. Revista Electrónica de Geografía y Ciencias Sociales. Universidad de Barcelona [ISSN 1138-9788]. № 21, 15 de mayo de 1998.

Pujol, Francesc, "Relligant Nou Barris", Ayuntamiento de Barcelona, 2003.

Serratosa, Albert, "La revisió del Pla Comarcal de 1953: Alternatives a l'origen i organització dels treballs", Paper n²8, Región Metropolitana de Barcelona, noviembre 1997, pag.9-14

Tatjer, Mercedes, "La vivienda obrera en españa de los siglos XIX y XX: De la promoción privada a la promoción pública (1853-1975)" Scripta Nova. Revista Electrónica de Geografía y Ciencias Sociales. Universidad de Barcelona. ISSN:1138-9788. Depósito Legal: B. 21.741-98 Vol. IX, núm. 194 (23), 1 de agosto de 2005

Fuentes documentales

Cartografía histórica 1933, Vicenç Martorell, @ Instituto de Cartografia de Catalunya

Ortofotoplano 1961, @ Mancomunidad de Municipios del Area Metropolitana de Barcelona

Ortofotoplano 1981, @ Mancomunidad de Municipios del Area Metropolitana de Barcelona 
Ortofotoplano 2010, @ Instituto de Cartografía de Catalunya

Plan de Ordenación de Barcelona y su zona de influencia, 1953

Plan General Metropolitano de Barcelona, 1976

Plan Especial de Reforma Interior del sector "Baro de Viver". Aprovación definitiva 23 de Abril de 1991.

Estudio de Detalle en el Barrio de Casas Baratas de "Eduard Aunós" Zona Franca. Aprobación definitiva 29 de Junio de 1990.

Estudio de Detalle en el Barrio de Casas Baratas de "Eduard Aunós". 1a y $2^{a}$ Fase. Aprobación definitiva 28 de Marzo de 1995.

Plan Parcial de remodelación del grupo de viviendas Milans del Bosch. Aprobación definitiva 17 de Diciembre de 1968.

Modificación Plan General Metropolitano en el polígono de las Casas Baratas del "Bon Pastor". Aprobación inicial 20 de Marzo de Plan Especial de Reforma Interior del sector "Can Carreras" y del parque de "Can Quintana". Aprobación inicial de 13 de Marzo de 1979.

Registro de planeamiento de Catalunya: http://ptop.gencat.net/rpucportal

Registro de planeamiento del Ayuntamiento de Barcelona: http://www.bcn.cat/urbanisme/

Federación de asociaciones de vecinos de Barcelona: http://www.favb.cat

Asociación de vecinos "Bon Pastor": http://www.bonpastor.org/

Plataforma repensar "Bon Pastor": http://repensarbonpastor.wordpress.com/

Asociación de vecinos "Can Peguera": http://www.9bacull.org/es/content/avv-can-peguera

Asociación de vecinos, grupo de memoria social: http://www.facebook.com/pages/LAS-CASAS-BARATAS-Eduard-Aunós

Planos históricos del transporte público en Barcelona: http://bcnmobilitat.wordpress.com/category/ historia/

Núria Noguer Pujadas, arquitecta

Estudiante del programa de Máster en Urbanismo (UPC) noguer.nuria@gmail.com

Adolf Sotoca Garcia, arquitecto. Doctor en Urbanismo (UPC) Profesor del programa de Doctorado en Urbanismo Departament d'Urbanisme i Ordenació del Territori. Universitat Politècnica de Catalunya adolf.sotoca@upc.edu 